\title{
Comparison of SCEM and STEM-HAADF Imaging in Thick Specimens
}

Nestor J. Zaluzec, Jon Hiller

Electron Microscopy Center, Argonne National Laboratory, Argonne, IL, USA 60439

The Scanning Confocal Electron Microscope has been shown to have significant advantages during imaging of thick specimens in both Materials and Biological applications. [1-4]. Since the electron optical configuration of STEM is a subset of the more general SCEM configuration, it is of interest to compare the performance of these two imaging modes. In the limit of infinitely thin specimens SCEM and STEM performance should be essentially be identical, this is born out by the "3D STEM" imaging of the ORNL group[5]. In this work we compare imaging at the other extreme.

A test specimen for analysis was fabricated by Focused Ion Beam (FIB) milling of a semiconductor device in a Zeiss $1500 \mathrm{XB}$ system. A periodic structure on the device was chozen so that resolution measurements could be made on nearly identical features as a function of thickness. Using the FIB, six flat neighboring parallel slabs were machined into the device having total thicknesses of 890, 1950, 2630, 3050, 3230 and $3870 \mathrm{~nm}$ (Fig. 1) measured by SEM imaging. After fabrication the specimen was mounted on a $3 \mathrm{~mm} \mathrm{Cu}$ slot grid and transferred to the ANL SCEM, which was operated at $300 \mathrm{kV}$ in both the SCEM and STEM-HAADF mode [2]. Adjustment between imaging modes was done by changing the currents in the $\mathrm{C} 1, \mathrm{C} 2, \mathrm{C} 3$, Objective and P1 lenses, the P2-P4 lenses of the instruments where not utilized in these experiments. In SCEM mode only C3 and P1 lenses were employed while in STEM-HAADF C1, C2, Obj, and P1 were employed. The specimen was oriented perpendicular to the incident probe and with the features of interest on the electron exit surface of the sample to maximize deleterious thickness effects.

Figure 2 presents unprocessed images from the thinnest $(890 \mathrm{~nm})$ and thickest $(3.87 \mu \mathrm{m})$ regions of this test specimen in both SCEM and STEM-HAADF modes. Identical regions of a flat $\mathrm{Cu}$ interconnect of width $0.8 \mu \mathrm{m}$ were imaged in each mode. In each case the dark level of the detector was always set to zero, however the gain was adjusted to $90 \%$ of saturation on the most electron transparent area of the specimen within the field of view. Figure 3 compares the normalized intensity profiles measured across the vertically oriented interconnect for the two extreme thicknesses. In all four profiles the average background intensity was subtracted and maximum intensity normalized. For display purposes the plot uses inverted contrast, and shows only the leading edge onset. The horizontal arrow in Fig $2 \mathrm{C}$ indicates the nominal position at which the profile was measured in each image. In the thinner region of the test specimen the resolution difference as measured by the signal rise profile width between SCEM $(\sim 44 \mathrm{~nm})$ and STEM-HAADF ( 49nm) is small, however we note that there is significantly more contrast available in the SCEM image due to the inclusion of diffraction contrast which is nearly absent in the STEM-HAADF image. In the 3.87 $\mu \mathrm{m}$ thick area, the profile resolution is clearly superior in SCEM $(\sim 125 \mathrm{~nm})$ vs. STEM-HAADF $(\sim$ $275 \mathrm{~nm}$ ) and is expected to be even greater in an aberration corrected SCEM.

References

[1] Frigo etal. , Applied Physics Letters Vol 81 \#11, 2112, (2002)

[2] Zaluzec N.J., Microscopy-Today Vol 6, 8 (2003). US. Patent \# 6,548,810 (2003)

[3] Hallegot P. and Zaluzec N.J., Microsc. Microanal. 7 Suppl. 2, 679 (2004).

[4] Gazda J. and Zaluzec N.J., Proceedings of ISSM 2004, Tokyo Japan, Oct. 2004 in press.

[5] Peng Y. etal Microsc. Microanal. 7 Suppl. 2 , 197 (2004). 
[6] This work was supported in part by the U.S. DoE under BES-MS W-31-109-Eng-38 at ANL
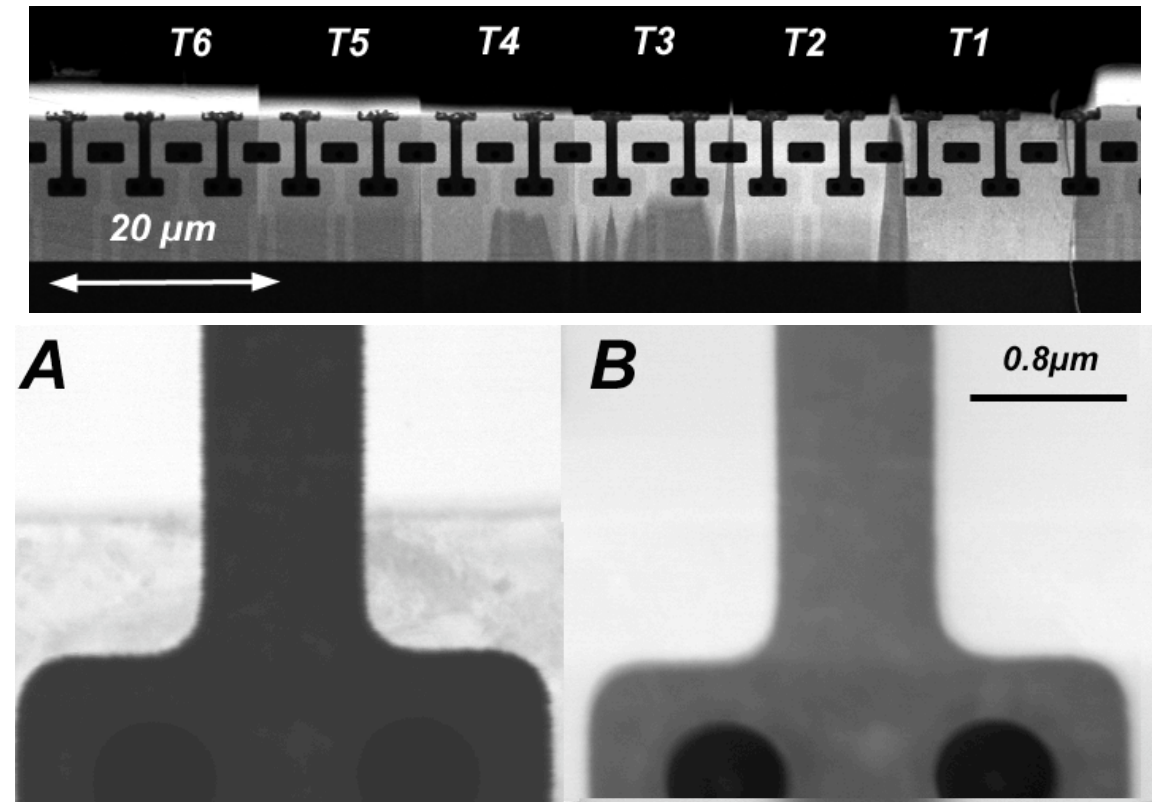

$0.8 \mu m$
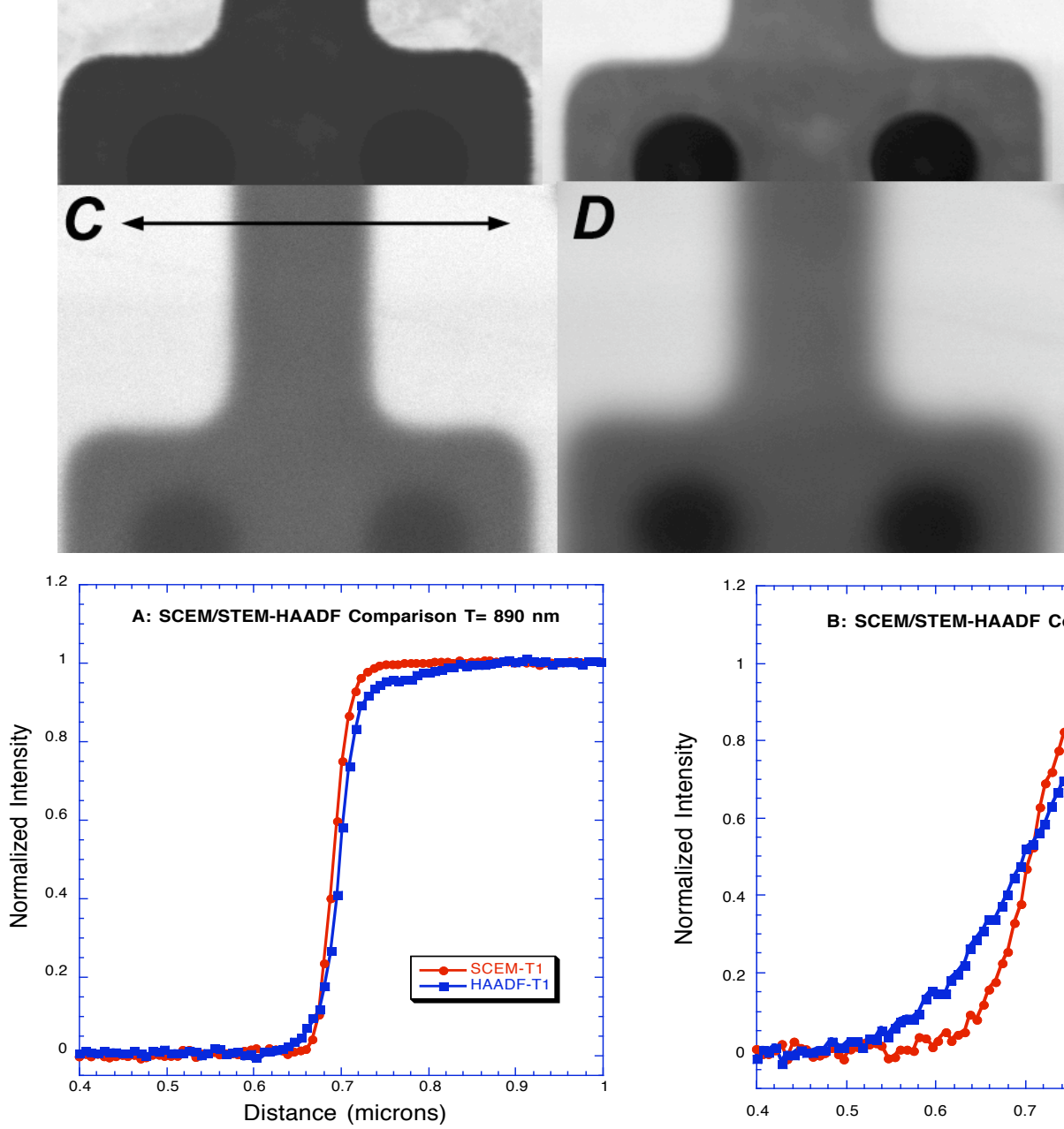

Figure 1:

Low Magnification image showing the test specimen and the 6 thickness zones. T1-T6 $=890,1950,2630$, 3050,3230 and $3870 \mathrm{~nm}$

Figure 2. Comparisons of $\operatorname{SCEM}(A, C)$ and STEM-HAADF (B,D) images in $890 \mathrm{~nm}$ $(\mathrm{A}, \mathrm{B})$ and $3870 \mathrm{~nm}$ (C,D) thick sections of a $\mathrm{Cu}$ interconnect in semiconductor device. The horizontal arrow in $\mathrm{C}$, indicates the positon in all images where the edge profile measurements were performed. 